\title{
LEAN MANUFACTURING COMO HERRAMIENTA DE COMPETITIVIDAD EN LAS PYMES ESPAÑOLAS
}

\section{LEAN MANUFACTURING AS A TOOL OF COMPETITIVENESS IN THE SPANISH SMES}

Miluska Aylin Añaguari Yarasca ${ }^{1}$

Víctor Gisbert Soler ${ }^{2}$

1. Ingeniera Industrial (Universidad de Lima - Perú). Máster Universitario de Ingeniería de la Organización y Logística (Universidad Politécnica de Valencia). E-mail: miaaya@epsa.upv.es

2. Doctor Ingeniero Industrial. Departamento de Estadística e Investigación Operativa Aplicadas y Calidad. Universidad Politécnica de Valencia. E-mail: vgisber@eio.upv.es 


\section{RESUMEN}

El presente artículo, en base a estudios previos y a la múltiple literatura escrita, tiene por objeto dar a conocer la importancia de la aplicación de las herramientas de la manufactura esbelta en las Pymes industriales españolas, mostrando cuales son los factores críticos que afectan directamente el desempeño competitivo de las pymes en la actualidad. Así también dar a conocer la metodología Lean Manufacturing, estrategias para su implementación y los beneficios que esta conlleva para la empresa.

\section{ABSTRACT}

This article, based on previous studies and multiple written literature, aims to raise awareness of the importance of the implementation of lean manufacturing tools in the Spanish industrial SMEs, showing which are the critical factors that directly affect the performance competitive SMEs today. As well as raise awareness of Lean Manufacturing, strategies for implementation and the benefits this brings to the company.

\section{PALABRAS CLAVE}

Pymes, manufactura esbelta, competitividad, despilfarro, valor añadido.

\section{KEY WORDS}

SMEs, lean manufacturing, competitiveness, wastage, value added. 


\section{INTRODUCCIÓN}

La globalización es un proceso dinámico de creciente libertad e integración mundial de los mercados de trabajo, bienes, servicios, tecnología y capitales. Peter Drucker sugiere que la manera más precisa de comprender la globalización es "desaprender lo aprendido" para generar un nuevo concepto que nos permita ampliar las fronteras, crear rupturas epistemológicas y generar nuevos conceptos [1].

Es aquí donde las empresas son llamadas al formar parte de este cambio constante. Actualmente, las Pymes, compañías pequeñas locales o regionales con el propósito de promover el desarrollo económico nacional que para ser llamadas así deben cumplir con ciertas características como: tener menos de 250 empleados contratados y por contratar, y no pueden otras organizaciones participar con más del $25 \%$ del capital accionario. Se enfrentan al reto de implementar nuevas técnicas organizativas y de producción que le permitan sobrevivir ante una competitividad incesante de las empresas, cambio en la mentalidad de los clientes e inestabilidad de la demanda.

Para ello se propone la filosofía Lean Manufacturing como una herramienta que permita a las pymes afianzarse como compañía analizando sus ventajas competitivas tomando partido de éstas para crear nuevos entornos de trabajo en base a una metodología nueva, que permita crear una cultura de cambio en la organización apoyado en el compromiso de la alta dirección y formación de los mandos medios y operarios buscando en todo momento la mejora continua en la organización.

\section{PYMES ESPAÑOLAS: SITUACIÓN ACTUAL}

Las pequeñas y medianas empresas (Pymes) son actualmente parte fundamental de la economía española y europea. Estas contribuyen decisivamente al crecimiento económico, creación de empleo y bienestar social. Según el Directorio Central de empresas (DIRCE), a 1 de enero del año 2013 hay en España 3.142.928 empresas de las cuales 3.139.106 (99.80\%) son Pyme (entre 0 y 239 asalariados), con un valor añadido bruto (VAB) por ocupado de la economía española de 54.251 euros los que representa una tasa de crecimiento interanual de $3.15 \%[2]$.

Así pues, el entorno actual se ve reflejado en el dinamismo en el cual las empresas deben moverse, la velocidad en los cambios, la inestabilidad de la demanda y la competencia tanto interna como externa; todo esto sustentado en la exigencia cada vez mayor de los clientes que requieren productos con los más altos estándares de calidad pero a precios asequibles, así como un servicio óptimo cantidades y tiempos requeridos.

En un análisis DAFO (fortalezas, debilidades, amenazas y oportunidades) se encontró como problemas relevantes que afrontan las Pymes lo siguiente [3]:

- Incapacidad para el crecimiento internacional. 
- Productividad y eficiencia en costes inferiores a la media de la competencia.

- Estrategias de precios pasivas o mercado - aceptantes.

- Desventaja en economías de escala y experiencia.

- Bajo potencial de diferenciación tecnológica basado en la innovación.

- Falta de liderazgo directivo.

- Escaso desarrollo de las competencias del capital humano y del aprendizaje.

- Debilidad de la posición financiera.

- Escasa o poca innovación en operaciones y procesos.

Muchos de estos problemas encontrados se encuentran directamente relacionados con el ámbito interno de la compañía, que pueden ser mejorables utilizando estrategias adoptadas por grandes corporaciones en la innovación de procesos como la metodología Lean Manufacturing, que consiste en la eliminación sistemática del desperdicio y asumiendo compromisos por parte de la dirección para que los resultados sean sostenibles a largo plazo.

\section{3. ¿QUÉ ES EL LEAN MANUFACTURING?}

El Lean Manufacturing es una metodología que tiene por objetivo la eliminación del despilfarro y maximizar o utilizar solo las actividades que añadan valor agregado desde la perspectiva del cliente, al producto o servicio final mediante la utilización de una colección de herramientas (TPM, 5S, SMED, kanban, kaizen, heijunka, jidoka, etc.), que se desarrollaron fundamentalmente en Japón, inspiradas en parte, en los principios de William Edwards Deming.

Los pilares del lean manufacturing son: la filosofía de la mejora continua, el control total de la calidad total, la eliminación del despilfarro, el aprovechamiento de todo el potencial a lo largo de la cadena de valor y la participación de los operarios. Está soportada en cinco principios básicos, que son: identificar y definir el valor (Value), identificar los flujos de valor (Value Stream), alinear las acciones de la organización con los flujos de valor (Flow), permitir que las necesidades y expectativas del clientes jalonen (Pull) y perseguir la perfección (Perfection) [3], [4].

Algunos de sus objetivos son:

- Defectos o desperdicios: Eliminarlos o minimizarlos, así como eliminar cualquier característica innecesaria del producto que no sea requerida por el cliente. Eliminar el exceso de materias primas y costos asociados por reprocesos.

- Tiempos de ciclo: Reducir plazos de entrega y ciclos de tiempo de producción mediante la reducción de tiempos de espera, de reparación o de preparación. 
- Niveles de inventario: Minimizar inventarios de materia prima, producto terminado y en todas las etapas de producción, especialmente los WIP (Trabajos en Proceso) entre las etapas de producción.

- Productividad laboral: Mejoramiento mediante la reducción de tiempo ocioso de los operarios. Asegurándose que su trabajo les produzca la satisfacción y beneficios que cumplan con sus expectativas en función de su involucración, resultados y participación en busca de la mejora continua.

- Utilización de equipos y de espacio: Usarlos de manera eficiente para eliminar cuellos de botella/restricciones. Maximizando el flujo y la velocidad de producción minimizando el tiempo de paro del equipo y maquinaria existente.

- Flexibilidad: Producir un rango y variedad más flexible de productos, con costos reducidos y tiempos mínimos de conversión. Empleando personal con polivalente, capacitado y entrenado continuamente; con capacidad para asumir responsabilidades conforme se le vaya cediendo autoridad y responsabilidad.

- Resultado: En la medida que se reduce el tiempo de ciclo, se incrementa la productividad laboral, se gestionan adecuadamente los cuellos de botella y restricciones. Lográndose costos de producción menores, uso más eficiente de la maquinaria y espacio.

\section{NECESIDADES PARA LA IMPLEMENTACIÓN DEL LEAN}

La filosofía Lean ha sido una estrategia para la mejora, por la velocidad con la que se obtienen los resultados, la escasa inversión necesaria y los beneficios reflejados en los costos. Para su implementación se deben analizar la situación actual de la empresa tal como productos, organización, objetivos a corto, mediano y largo plazo, procesos de producción, etc. Sumado a esto hay que considerar la implicación de toda la empresa en su implantación desde el compromiso de la alta dirección que debe invertir recursos suficientes; una pieza clave en una implantación exitosa es el Factor del Entorno especialmente el Factor Humano el cual debe ser formado en cultura Lean [2], [3].

A continuación, se presentan los obstáculos más frecuentes que pueden determinar el curso del proyecto. 


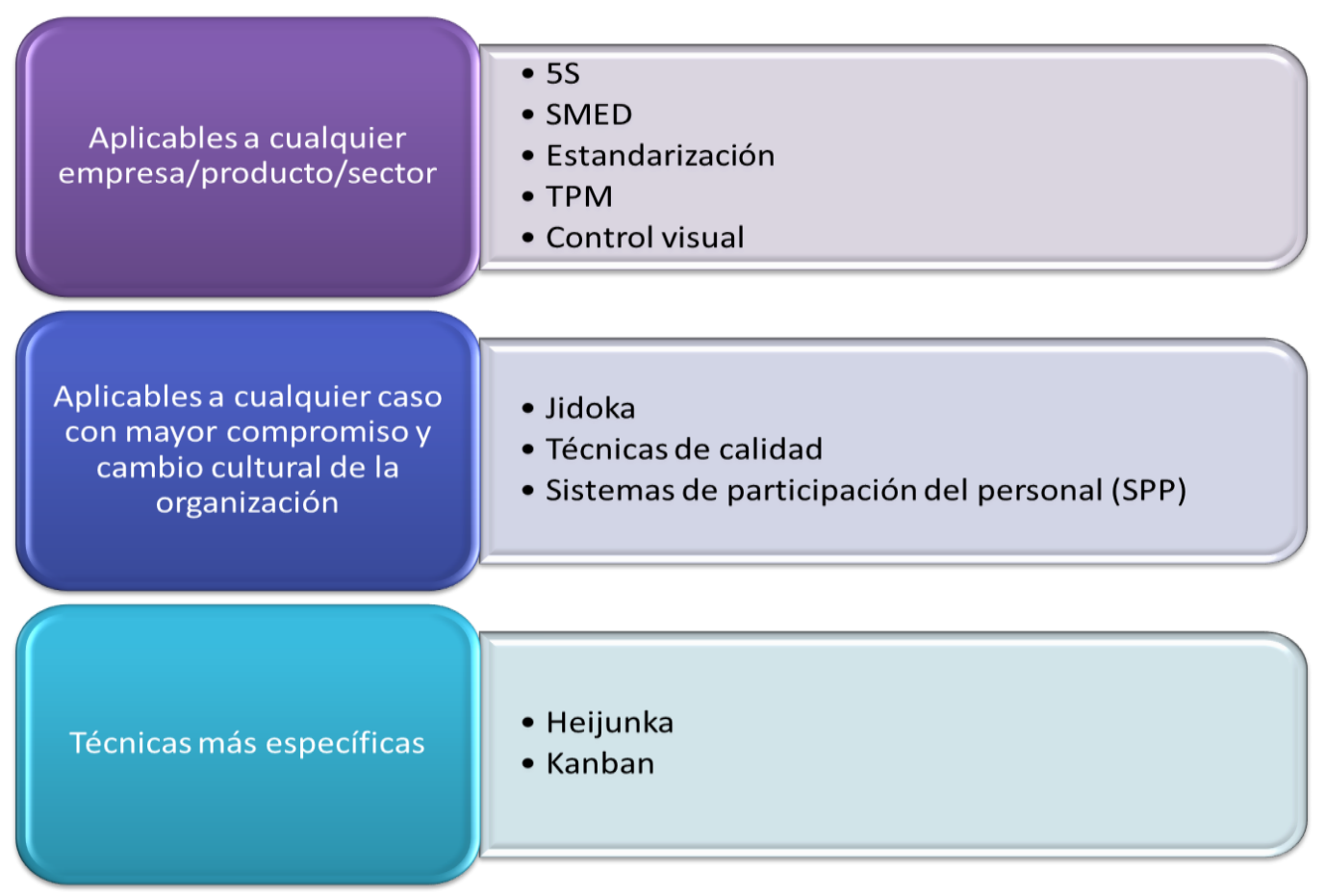

Gráfico 3: Técnicas del Lean Manufacturing. Fuente: Elaboración Propia.

Para implementar cualquiera de estas técnicas se requiere el compromiso de la empresa en invertir en formación del personal, pieza clave en la implementación, como ya lo hemos dicho, así como promover la cultura de la mejora continua.

Se recomienda seguir la siguiente hoja de ruta, la cual está constituida por una posible secuencia de fases y elementos que permitan a las empresas diseñar el mejor camino para una implementación Lean Manufacturing.

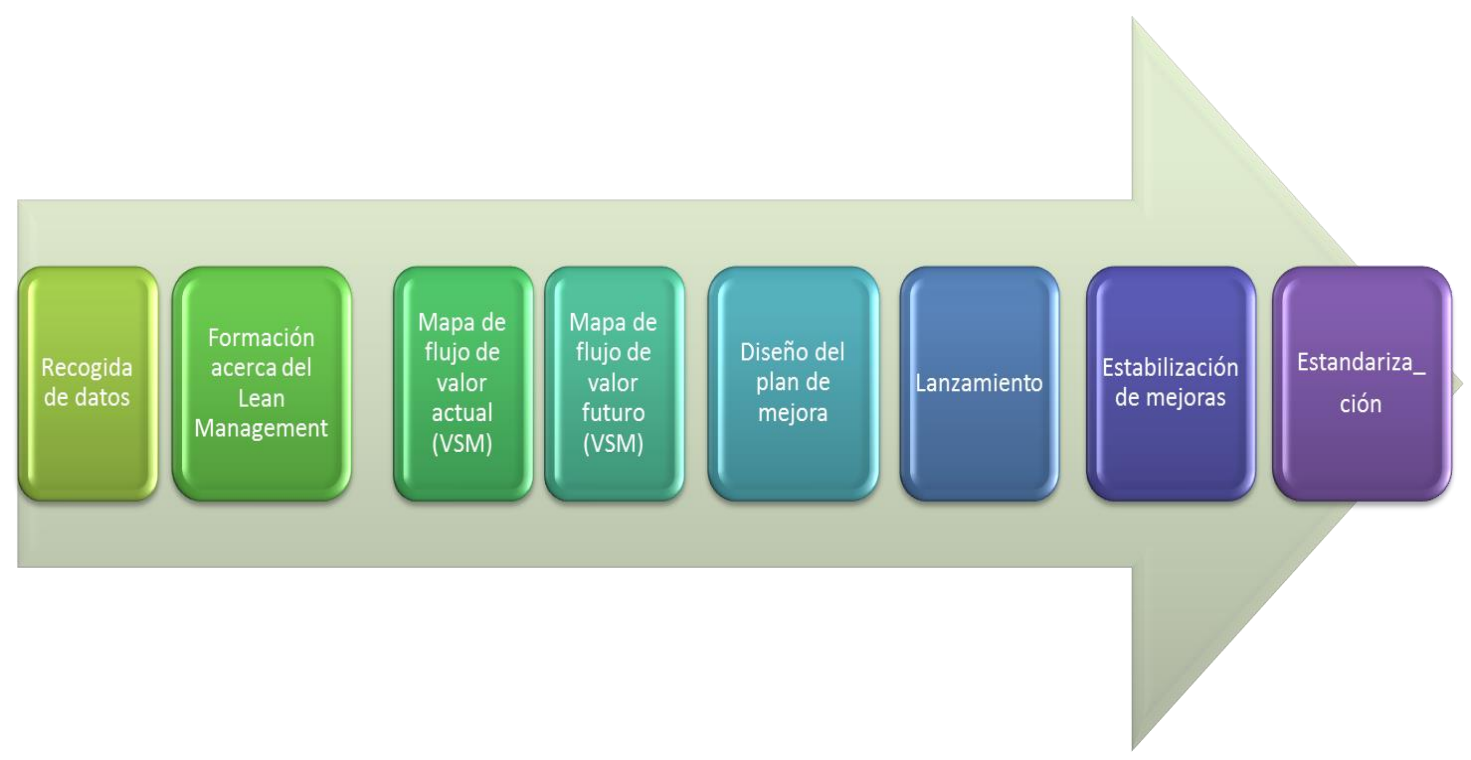

Gráfico 4: Pasos para la implementación de Lean Manufacturing.

Fuente: elaboración propia. 


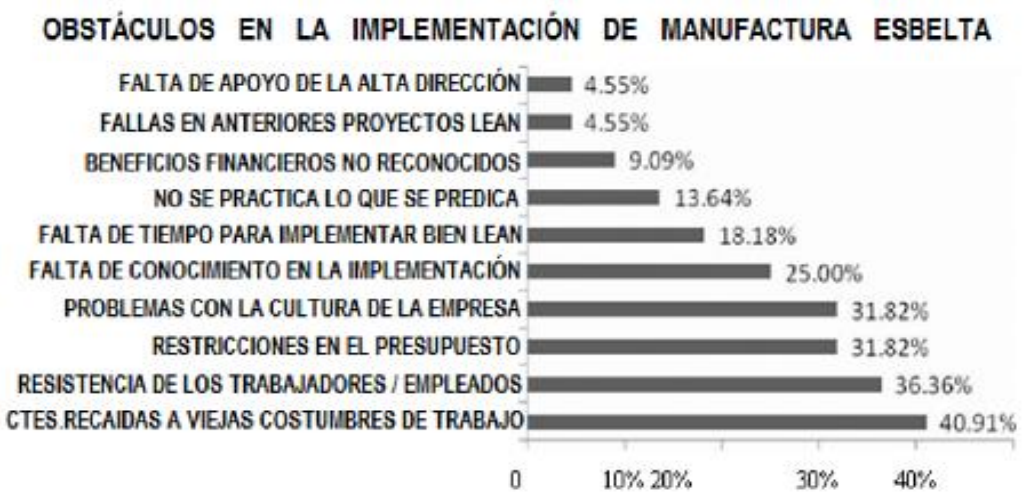

Gráfico 1: Obstáculos en la implementación de la manufactura esbelta.

Fuente: Yu Cheng Wong, Kuan Yew Wong \& Anwar Ali (2009).

De forma tradicional, se ha recurrido al esquema de la "Casa del Sistema de Producción Toyota" ya que en él se visualiza fácilmente la filosofía que encierra el Lean y las técnicas disponibles para su aplicación. Aquí se identifican las metas que persigue la organización como son mejor calidad, bajo costo, tiempos de entregas menores, apoyado en los dos pilares fundamentales del sistema: JIT y Jidoka [4]. La base consiste en la estandarización y estabilidad de los procesos: el heijunka o nivelación de la producción y la aplicación sistemática de la mejora continua. Sumándose a ello el factor humano como clave para la implementación del Lean. Estos elementos se aplican a través de múltiples técnicas que hablaremos a continuación. 


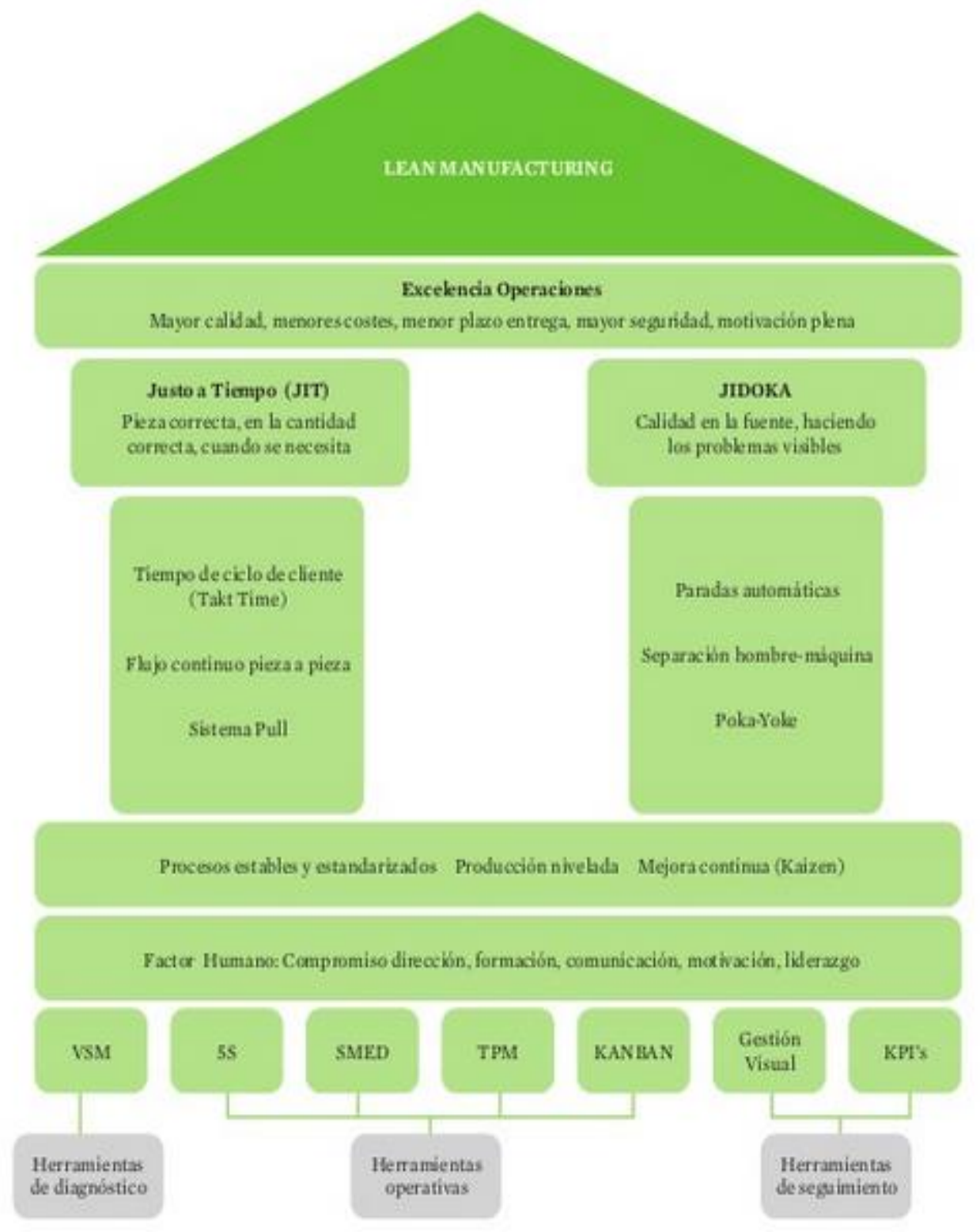

Gráfico 2: Adaptación actualizada de la casa Toyota.

Fuente: Escuela de Organización Industrial (2013).

\begin{tabular}{|l|llll|}
\hline 5. TÉCNICAS & Y & HERRAMIENTAS & DE & LEAN \\
MANUFACTURING & & & & \\
\hline
\end{tabular}

La manufactura esbelta se pone en práctica a través de la aplicación de una amplia variedad de técnicas que se han ido implementando con éxito en compañías de diferentes tamaños y sectores. Éstas pueden aplicarse de forma conjunta o independientemente, se recomienda que se haga de forma secuencial adaptándose a la realidad particular de cada caso, equilibrando esfuerzos y recursos con los objetivos de mejora propuestos y la realidad. 


\section{CONCLUSIONES}

En base a todo lo expuesto anteriormente, se puede concluir que las técnicas y herramientas Lean usadas pueden ayudar a mejorar la competitividad de la empresa siempre y cuando se escoja un Plan de Implementación adecuado a las condiciones reales de la empresa teniendo en cuenta los objetivos que se quieren cumplir reflejados en indicadores claves de producción que permitan medir si el proceso está mejorando.

Así también recalcar el compromiso de toda la organización desde la alta dirección con la dotación de recursos así como del personal el cual debe estar formado y concientizado de los cambios que se pueden lograr y qué su participación es clave en el proceso de cambio.

Una vez que se haya implementado el sistema Lean es vital la mejora continua buscando pequeños cambios que puedan hacer la diferencia en los procesos productivos centrándonos en la eliminación de actividades que no generen valor y potenciando aquellas que si todo esto con el fin de mejorar la competitividad de la compañía aumentando los márgenes de ganancia y reduciendo costes de fabricación. 


\section{REFERENCIAS BIBLIOGRÁFICAS}

[1] Camisón, C. y De Lucio, J. (2010). La competitividad de las pymes españolas ante el reto de la globalización. Revista Economía Industrial, 375(1), 19 -40. [Consulta: 19 junio del 2015]. ISSN 0422-2784. Recuperado de:

$<$ http://www.minetur.gob.es/Publicaciones/Publicacionesperiodicas/Economialndust rial/RevistaEconomialndustrial/375/19.pdf7/>.

[2] Feld, W.M. (2000). Lean Manufacturing: Tools, Techniques, and How to Use Them. Florida: St. Lucie Press. [Consulta: 29 de mayo del 2015]. Recuperado de: <https://books.google.com.pe/books?id=zM qqlrHKJ8C\&printsec=frontcover\&dq=le an+manufacturing\&hl=es419\&sa=X\&ei=IwKLVeqTGInlUay9gYgB\&ved=0CEUQ6AEwA

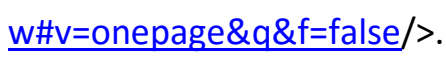

[3] Hernández Matías, J.C. y Vizán Idolpe, A. (2013). Lean Manufacturing: conceptos, técnicas e implantación. Madrid: Escuela de Organización Industrial, Universidad Politécnica de Madrid. [Consulta: 20 de junio del 2015]. Recuperado de: $<$ http://es.slideshare.net/slides eoi/lean-manufacturing-conceptos-tcnicas-eimplantacin/>.

[4] Rajadell Carreras, M. y Sánchez García, J.L. (2010). Lean Manufacturing. La evidencia de una necesidad. Madrid: Ediciones Díaz Santos. [Consulta: 15 de junio del 2015]. Recuperado de: $<$ https://books.google.com.pe/books?id=mZCh1a3L8M8C\&printsec=frontcover\&dq=I ean+manufacturing\&hl=es419\&sa=X\&ei=IwKLVeqTGInlUay9gYgB\&ved=0CDEQ6AEw AA\#v=onepage\& $q=$ lean $\% 20$ manufacturing $\& f=$ false $/>$.

[5] Subdirección General de Apoyo a la PYME. (2014). Retrato de las Pymes 2014. Madrid: Dirección General de la Industria y de la Pequeña y Mediana Empresa. [Consulta: 14 de junio 2015]. Recuperado de:

<http://www.ipyme.org/Publicaciones/Retrato PYME 2014.pdf/>. 